\title{
Colgajo frontal paramediano de pedícullo estrecho en reconstrucccion de cubierta nasal total: un caso interesante
}

\section{Forehead paramedian flap with narrow pedicle for total skin surface of the nose: an interesting case report}

Jezabel DE ABULLARADE*

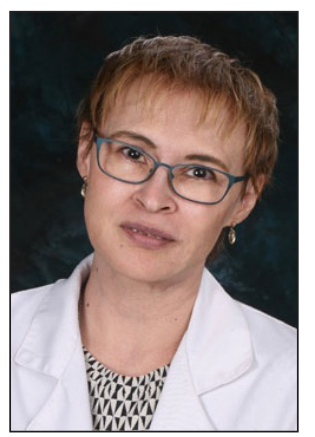

De Abullarade, J.

\section{Resumen}

Son pocos los casos de defecto total de la piel de la nariz al extirpar carcinomas no melanocíticos cutáneos.

Con este trabajo queremos conocer la seguridad de la aplicación del colgajo frontal paramediano de pedículo estrecho en un paciente de edad avanzada.

Aplicamos la técnica en 3 tiempos quirúrgicos para la reconstrucción de la cubierta de piel nasal. El defecto del área donante cerró directamente. No se presentó necrosis del colgajo ni infección y el resultado fue estético y funcional.

Valoramos así la seguridad de la reconstrucción de la cubierta total de piel de la nariz en pacientes de edad avanzada con el colgajo frontal paramediano de pedículo estrecho en 3 tiempos quirúrgicos.
Palabras clave Colgajo frontal, Colgajo frontal paramediano Nariz, Reconstrucción nasal,

Carcinoma basocelular.

Nivel de evidencia científica Recibido [esta versión] Aceptado
$4 \mathrm{~d}$ Terapéutico

3 febrero/2018

4 marzo/2019

Conflicto de intereses: los autores declaran no tener ningún interés financiero relacionado con el contenido de este artículo. Financiación: No hubo fuentes externas de financiación para este trabajo.

Total resection of the skin of the nose in non-melanoma skin carcinoma is rare.

In this paper we want to know the safety of the performance of the forehead paramedian flap with narrow pedicle in an elderly patient.

Three stage forehead paramedian flap with narrow pedicle was performed in the lining reconstruction of the total skin nasal surface. The donor site was closed primarily. Necrosis of the flap did not occur, nor infection happened. Excellent aesthetic and functional results were achieved.

We conclude on the safety of the reconstruction of the total surface of the skin of the nose in the elderly patient with the three-stage forehead paramedian flap with narrow pedicle.

$\begin{array}{lr}\text { Key words } & \begin{array}{l}\text { Forehead flap, Forehead paramedian flap, } \\ \text { Nose, Nasal reconstruction, } \\ \text { Non-melanoma skin cancer. }\end{array} \\ & \\ \text { Level of evidence } & 4 \mathrm{~d} \text { Therapeutic } \\ \text { Received [this version] } & 3 \text { February/2018 } \\ \text { Accepted } & 4 \text { March/2019 }\end{array}$




\section{Introducción}

Los defectos de espesor completo más frecuentes de la cubierta cutánea de la nariz son parciales y pueden ser reconstruidos con colgajos locales ${ }^{(1,2)}$ que plantean al cirujano reconstructivo el reto de no dejar un efecto tipo parche en el resultado final, pues no olvidemos que nuestra finalidad como especialistas es la de reponer los tejidos pero de una manera tal que camuflaje el defecto, recobrando la cobertura con un fin funcional y estético sin importar la edad del paciente mientras su estado de salud lo permita y respetando los principios de las subunidades nasales. ${ }^{(3)}$ Sin embargo, algunas veces nos enfrentaremos a casos poco frecuentes en los que el defecto de espesor completo de la piel es de toda la cubierta de la nariz, como en el caso que deseamos presentarles.

Está demostrado que a medida que utilizamos las cubiertas de piel regionales en relación a las distantes, logramos un mejor resultado estético y funcional facial, ${ }^{(4)}$ Así pues, para nuestro caso, en el que la afectación era de toda la cubierta de piel total nasal, la mejor opción de reconstrucción estética y funcional descrita entre las cubiertas de piel regionales es la piel de la frente, ${ }^{(5)}$ debido a que su espesor y características propias la hacen ser la que más se asemeja a la piel de la nariz.

En una publicación anterior en esta misma revista, describimos didácticamente la modificación del colgajo frontal paramediano con pedículo estrecho basada en la seguridad de la constancia anatómica de la irrigación y drenaje por la arteria supratroclear en el borde orbitario, demostrando sus ventajas en relación con el colgajo clá$\operatorname{sico}^{(6)}$ en reconstrucciones de subunidades. Ahora bien, quisiéramos introducir la modificación de pedículo estrecho del colgajo frontal paramediano en la reconstrucción del espesor completo de piel de la totalidad de la cubierta nasal, agregando que la realizamos, en este caso, en un paciente de edad avanzada, y conociendo que esta población, además de otros cambios anatomo-fisiológicos que afectan a todo el organismo, sufre cambios en los capilares que se engruesan ligeramente, pudiendo ocasionar disminución en la microcirculación. A pesar de ello, presentamos su ventaja y seguridad al levantarlo en 3 tiempos quirúrgicos, ${ }^{(7)}$ con un pedículo tan estrecho.

Describimos a continuación en detalle el caso y la técnica quirúrgica empleada.

\section{Caso clínico}

Tuvimos la oportunidad de aplicar la técnica del colgajo frontal paramediano de pedículo estrecho en un paciente de 74 años de edad, a quien en varias ocasiones el mismo cirujano le había extirpado focos de carcinoma basocelular en diferentes partes de la nariz, con respuesta de las respectivas biopsias por parafina de limites quirúrgicos con afectación positiva y por ende, con recidiva del carcinoma en toda la piel de la nariz.

Entre sus antecedentes clínicos presentaba hipertensión arterial controlada desde hace 20 años, sin antecedentes cardiacos ni diabetes, no tabaquismo ni enfermedades bronquiales, ni uso de anticoagulantes.

En el primer tiempo quirúrgico, bajo anestesia general, extirpamos toda la piel nasal afectada por el carcinoma basocelular residual en dorso, paredes laterales, fosa triangular, punta y alas nasales (Fig. 1) y enviamos la pieza a Patología para biopsia por congelación. El informe reportó bordes quirúrgicos sanos, por lo que procedimos a diseñar, levantar e interpolar el colgajo frontal paramediano de pedículo estrecho descrito por nosotros. ${ }^{(6)}$ En este mismo tiempo quirúrgico aplicamos puntos para

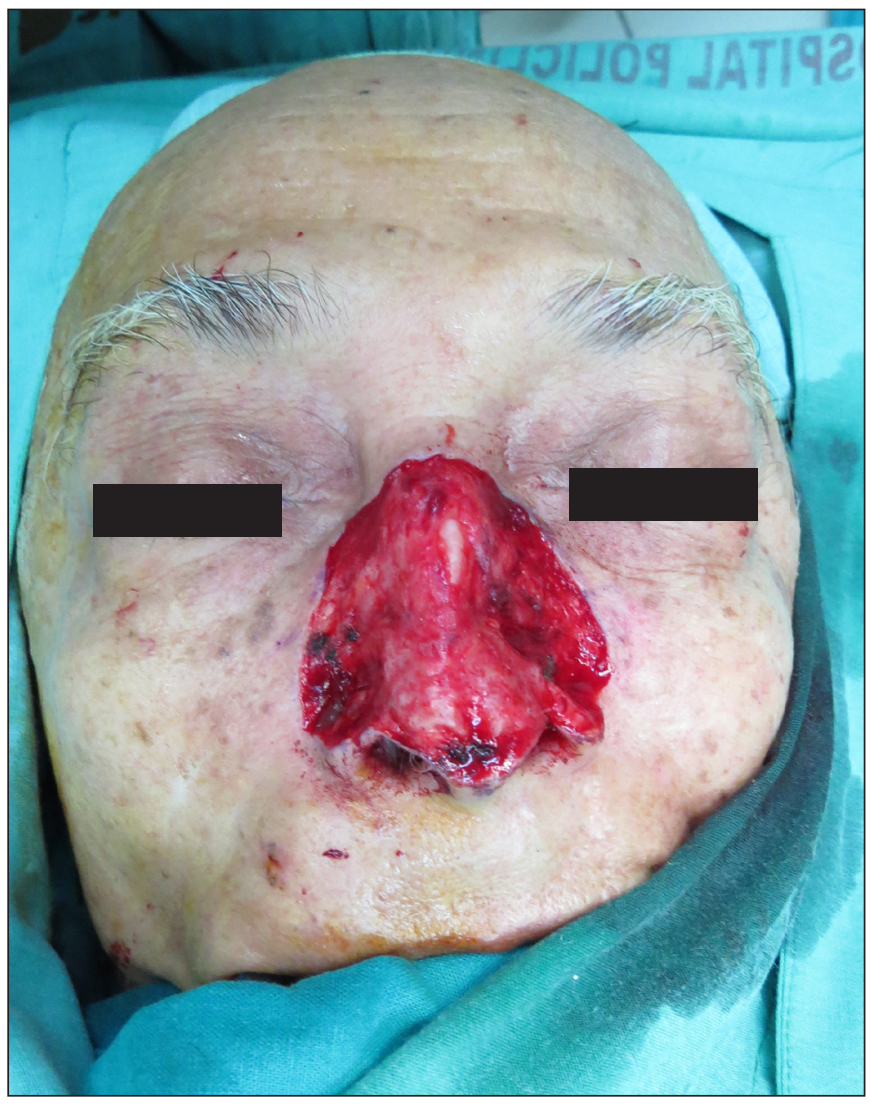

Fig. 1. Límites quirurgicos negativos en paciente de 74 años con reseccion completa de la piel nasal por carcinoma basocelular.

moldear los bordes de las alas nasales, que mantuvimos durante 6 semanas para asegurar el pliegue de contorno natural de esa área; estos puntos no afectaron en ningún momento a la circulación del colgajo, a pesar de su pedículo estrecho. El cierre del defecto donador en la frente fue directo, quedando un pequeño defecto remanente que cerró por segunda intención con resultados estéticos y funcionales satisfactorios (Fig. 2).

El tiempo operatorio fue de 3 horas y media. Al pa- 


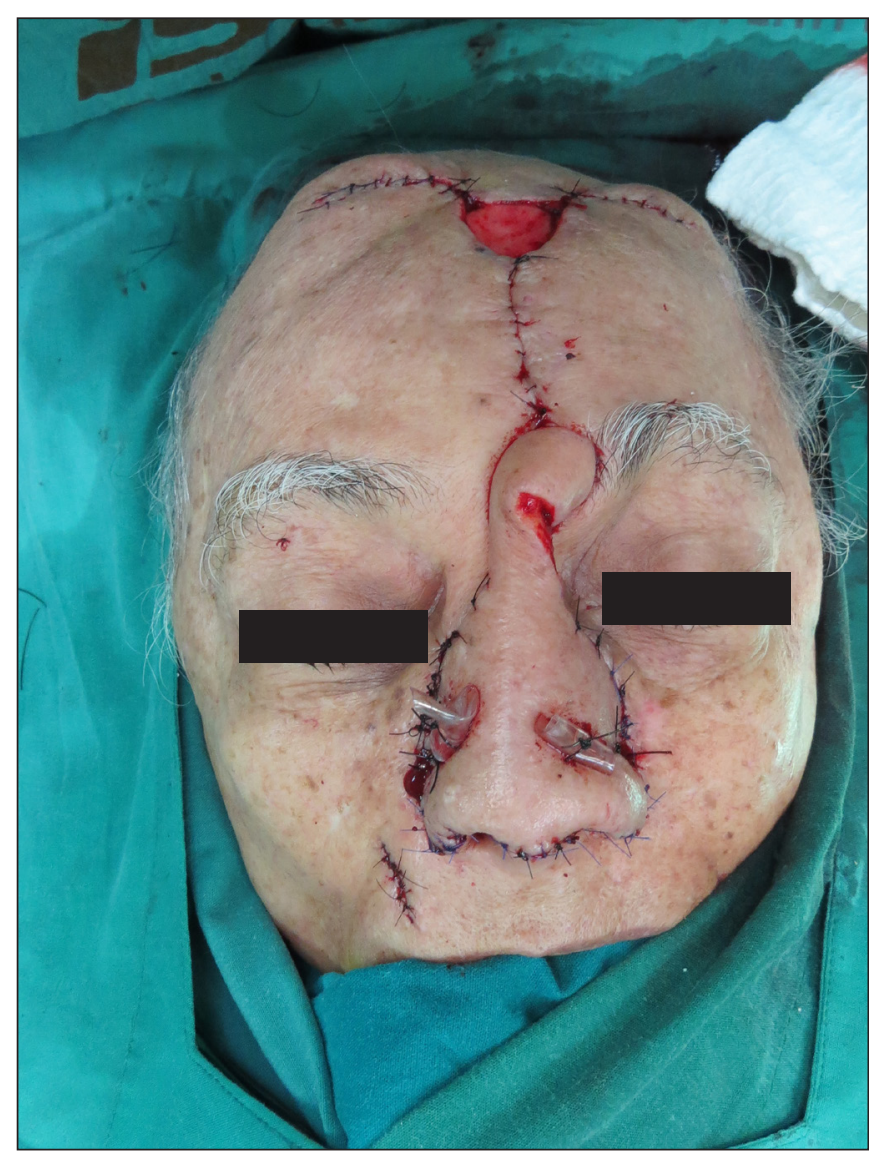

Fig.2 Reconstruccion imediata con colgajo frontal paramediano de pedículo estrecho para defecto de piel total de la nariz. Cierre directo de la zona donante y defecto remanente en la misma. Integridad del colgajo de pedículo estrecho del colgajo a pesar de los puntos para moldear las alas nasales.

ciente se le despertó de la anestesia general sin ninguna complicación que reportar, y se le cubrió con antibiótico endovenoso durante 3 días, tiempo que estuvo ingresado en el Servicio, siendo dado de alta sin ninguna novedad.

En el segundo tiempo quirúrgico, 3 semanas después del primero y bajo anestesia local con sedación, levantamos el colgajo bipediculado para adelgazar el dorso, paredes laterales y fosa triangular, manteniendo el pedículo proximal en los vasos supratrocleares y el pedículo distal en la punta y alas nasales y conservando los puntos dados para moldear los contornos nasales. En esta ocasión la cirugía duro 45 minutos y sin incidentes, por lo que el paciente fue manejado en régimen ambulatorio (Fig. 3 y 4).

El tercer tiempo quirúrgico lo realizamos 3 semanas después del segundo, y en él, bajo anestesia local y sedación, realizamos autonomización de su pedículo proximal en los vasos supratrocleares y retirada de los puntos de moldeamiento de las alas nasales. El tiempo operatorio empleado fue de 20 minutos, también sin complicaciones y en régimen ambulatorio.

No observamos durante el postoperatorio complicaciones como infección o sufrimiento o necrosis del colgajo. El paciente fue colaborador durante todo el proce-

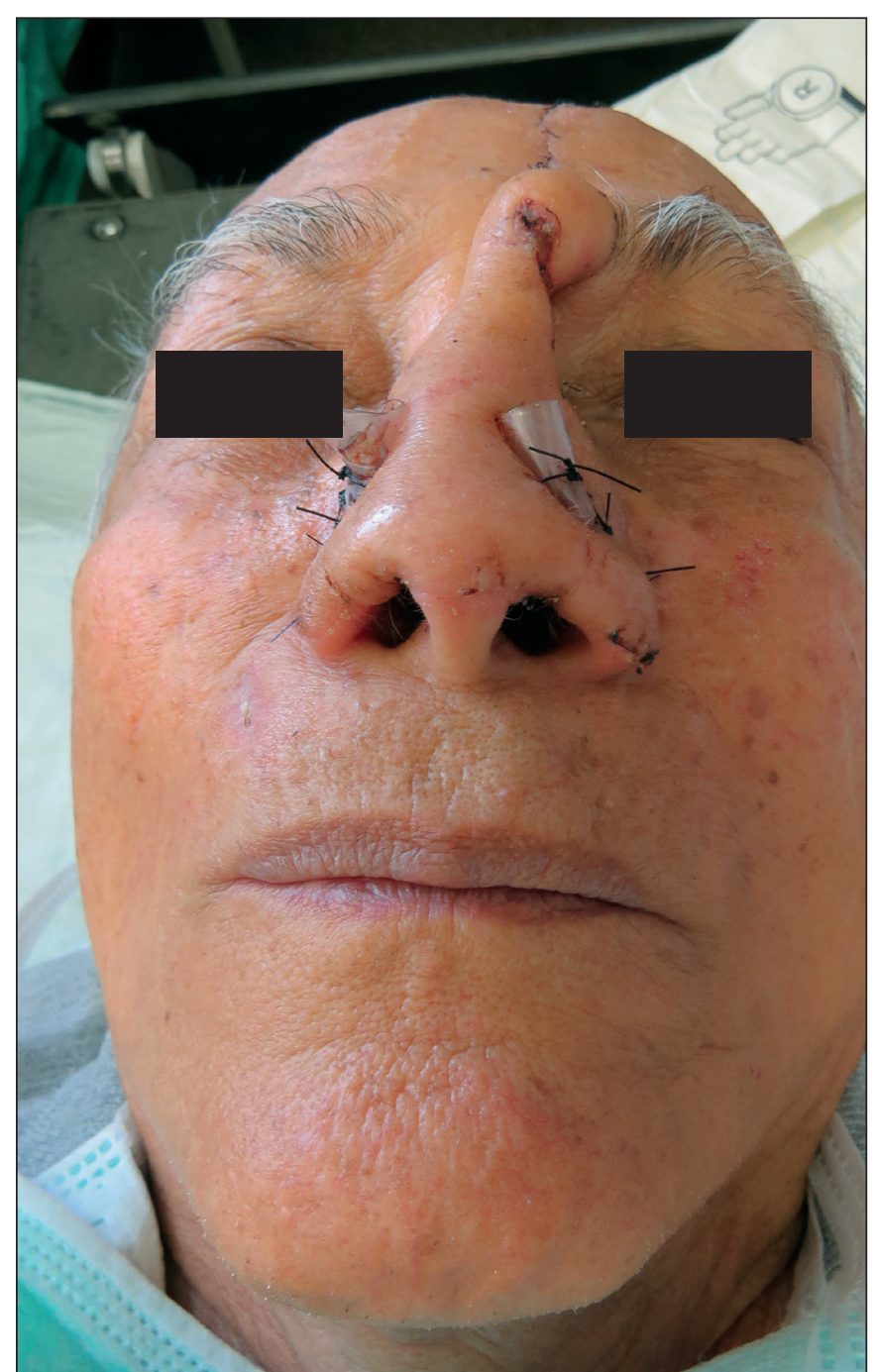

Fig. 3. Reconstruccion a las 3 semanas de postoperatorio

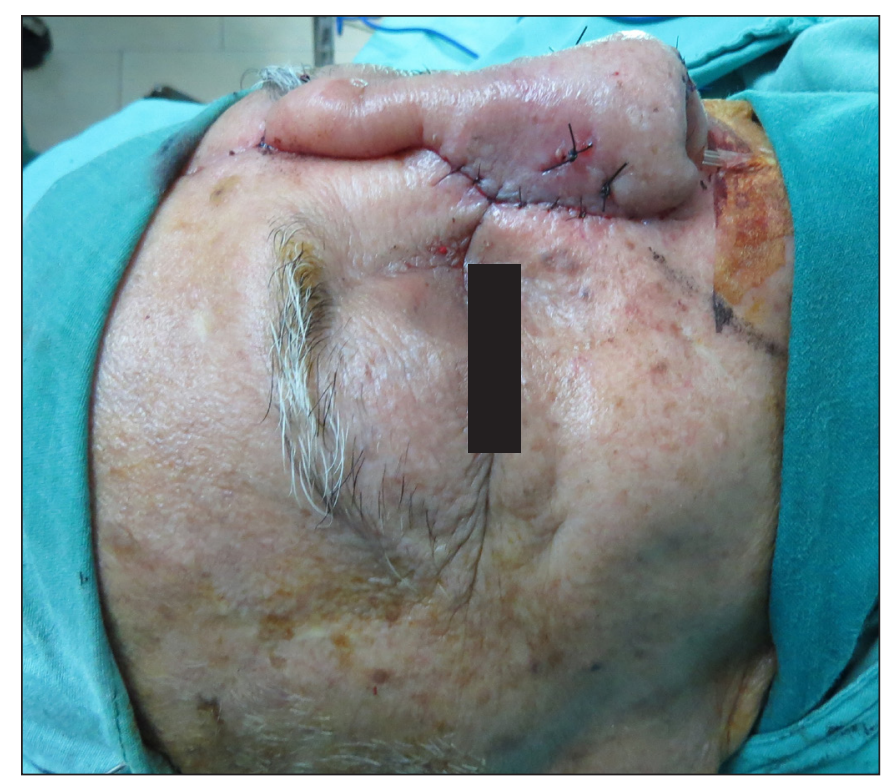

Fig. 4 Vista inmediata tras el adelgazamiento del colgajo.

so, atendiendo todas nuestras indicaciones de cuidados y se mostró muy satisfecho con los resultados (Fig. 5-7). En la actualidad sigue controles cada 6 meses (Fig. 8), y tras 1 año de seguimiento, no presenta complicaciones ni recidivas. Está previsto un seguimiento a 5 años. 

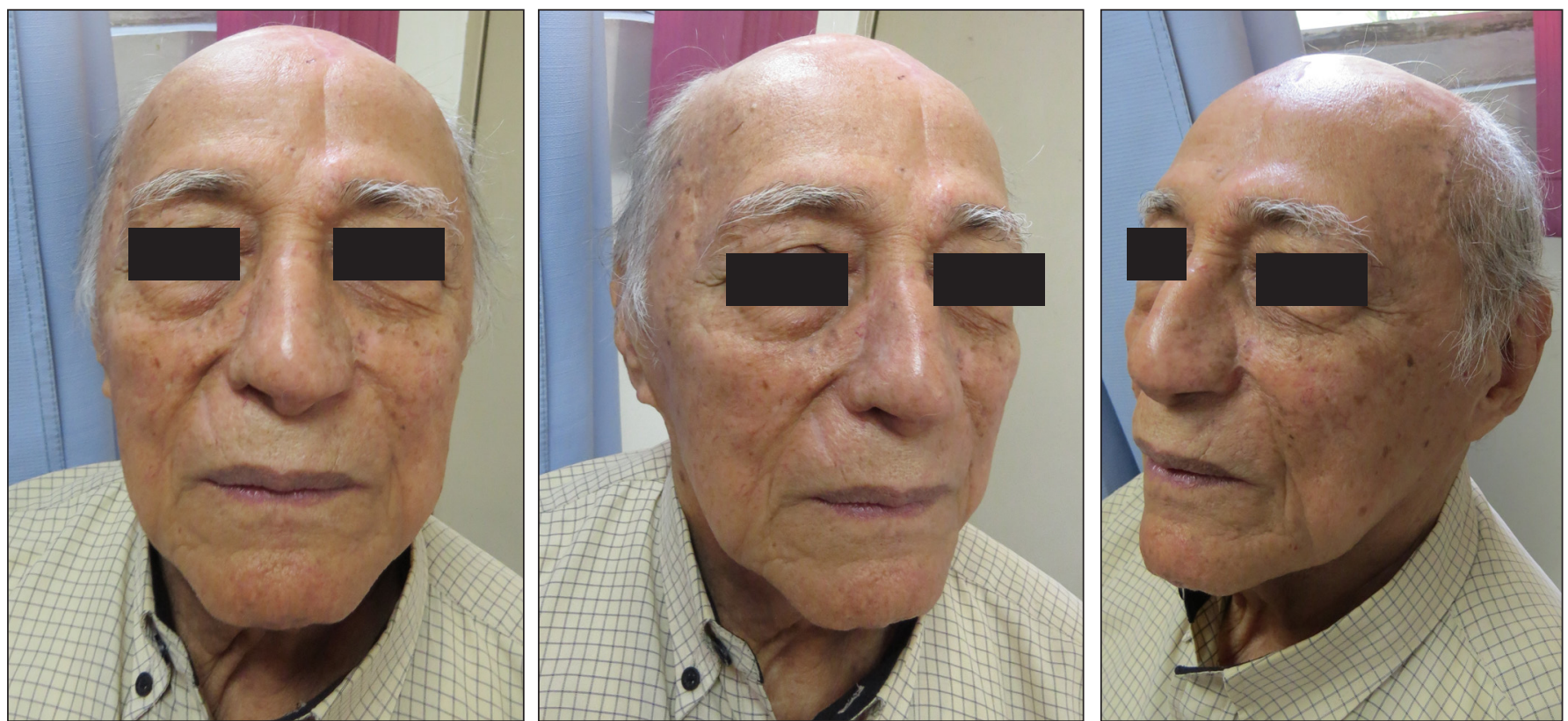

Fig. 5-7. Resultado a los 5 meses de postoperatorio. Excelentes resultados estéticos y funcionales de la zona donante y de la receptora.

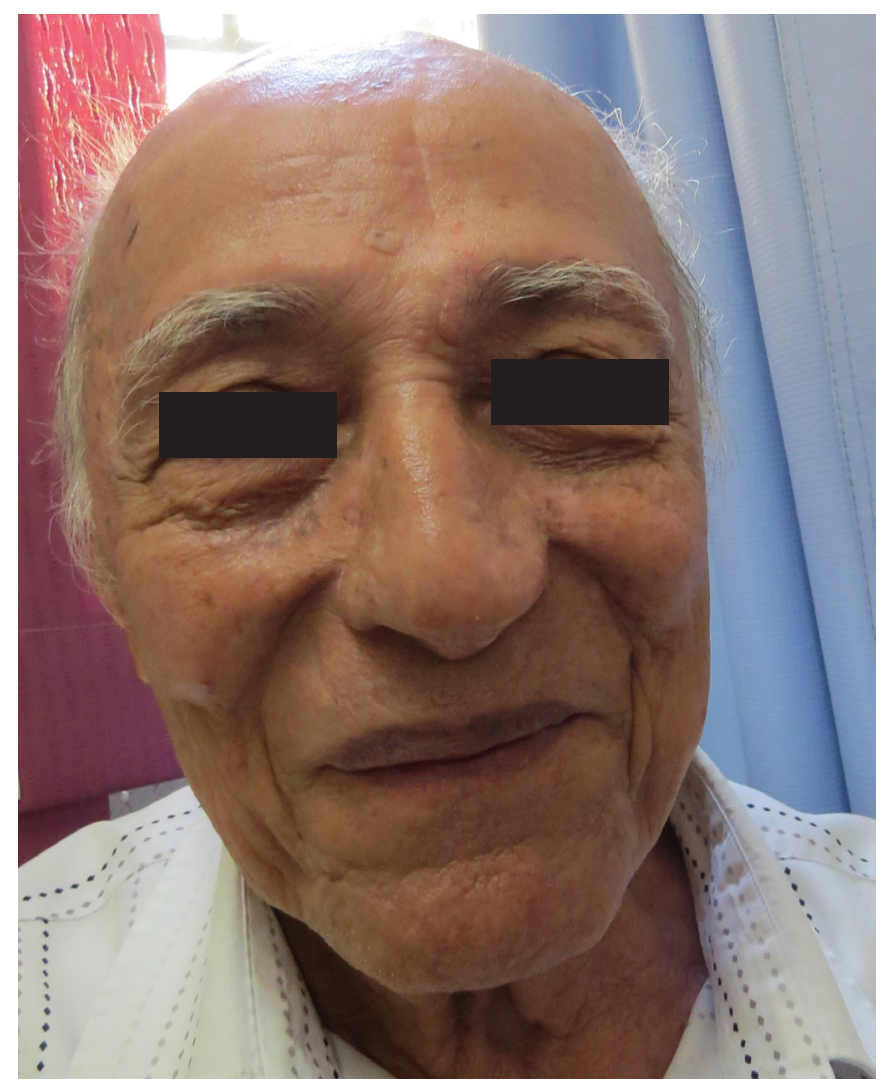

Fig. 8. Vista frontal del postoperatorio a 1 año de evolución.

\section{Discusión}

El colgajo frontal es una de las principales herramientas de todo cirujano plástico para la reconstrucción de la cubierta de espesor completo de la nariz, asumiendo que el paciente desea verse normal y mantener su funcionalidad y estética ${ }^{(5)}$ aunque tenga enfermedades de base. No es usual que por ese motivo se le deje cierre por segunda intención o se le coloque un injerto inestético, porque el objetivo de todo cirujano reconstructivo será el obtener un buen resultado y a ser posible, evitar grandes cirugías que incremente su morbimortalidad..$^{(7)}$

El colgajo frontal paramediano de pedículo estrecho(6) es factible en pacientes de avanzada edad si su estado de salud general lo permite, razón por la cual decidimos aplicarlo en el paciente que presentamos ya que gozaba de un estado de salud estable, si bien por su edad avanzada y por los cambios fisiológicos a nivel capilar que sabemos que se producen a esa edad, preferimos realizar la técnica en 3 tiempos quirúrgicos para garantizar la integridad del resultado.

La piel de espesor total de la frente es gruesa y firme, similar a la piel de la nariz en todas sus características, pero por su firmeza y grosor es necesario remodelarla hacia los contornos en 3 dimensiones de la estructura nasal. La necrosis de este colgajo frontal está descrita en pacientes fumadores y en pacientes de reconstrucción total de piel nasal ${ }^{(8)}$ debido a este riesgo, el Dr. Frederick J. Menick demostró la utilidad de mantener la seguridad de dicho colgajo realizándolo en 3 tiempos quirúrgicos en este tipo de pacientes

En nuestro caso, elegimos colocar desde el inicio puntos en los bordes de las alas nasales para moldear sus 3 dimensiones, y en un segundo tiempo adelgazar el colgajo en las paredes laterales y el dorso nasal, respetando la punta y las alas nasales a modo de colgajo bipediculado, comprobando que el colgajo estrecho es seguro de levantar (toda su cubierta nasal de piel de espesor completo en dorso, paredes laterales y fosa triangular) en un segundo tiempo quirúrgico para adelgazarlo a las 3 semanas de la primera intervención. Al hacer esto provocamos un fenómeno de retardo, tal y como explica 
Burguet, ${ }^{(9)}$ formando un colgajo bipediculado con suplemento vascular proximal por parte de la arteria supratroclear y distal en su porción de la punta de la nariz, en donde en ningún momento del proceso de adelgazamiento llevamos a cabo despegamiento. Este fenómeno de retardo asegura este paso en colgajos frontales de pedículo estrecho en la totalidad de la cubierta nasal, que a pesar del gran tamaño del colgajo levantado que queda sostenido por un pedículo tan estrecho, no presentó sufrimiento parcial, ni de los bordes, ni al adelgazarlo a las 3 semanas para su remodelación; tampoco al cortar su pedículo vascular en un tercer tiempo quirúrgico a las 3 semanas del segundo y tratándose de un paciente de edad avanzada.

Está demostrado que el colgajo frontal paramediano de pedículo estrecho es más manejable en la interpolación y tolera cierta tensión hacia lugares más distales, como la columela, que el colgajo frontal clásico, ventaja que se aplica también si el colgajo se levanta tanto para un defecto parcial como total de la nariz.

\section{Conclusiones}

El colgajo frontal paramediano de pedículo estrecho es seguro para dar cobertura total de piel en reconstrucción nasal en personas de avanzada edad utilizando el método de 3 tiempos quirúrgicos, a fin de garantizar la integridad del resultado.

\section{Dirección del autor}

Dra. Jezabel de Abullarade

Servicio de Cirugía Plástica del I.S.S.S.

Unidad Médica Atlacatl-Hospital Roma

Blvd. Venezuela y C. El Progreso, Colonia Roma

San Salvador, El Salvador, Centroamérica.

Correo electrónico: drdeabullarade@gmail.com

\section{Bibliografía}

1. De Abullarade J. Versatilidad del Colgajo Cigomático de Mejilla en la Reconstrucción Nasal. Cir. plást. iberolatinoam. 2012, 38 (3):273-277.

2. Pérez, M. et al. Aplicaciones del Colgajo Frontonasal para la Cobertura de Defectos Nasales. Cir. plást. iberolatinoam. 2015, 41 (4): 419-425.

3. Burget, G.C. and Menick, F.J. The Subunit Principle in Nasal Reconstruction. Plast. Reconstr. Surg., 1985. 76(2): 239-247.

4. Menick, F.J. Facial Reconstruction with Local and Distant Tissue: The Interface of Aesthetic and Reconstructive Surgery. Plast. Reconstr. Surg., 1998, 102(5):1424-1433.

5. Menick, F.J. Nasal Reconstruction: Forehead Flap. Plast. Reconstr. Surg., 2004, 113(6): 100e-111e.

6. De Abullarade, J. Colgajo Frontal Paramediano de Pediculo Estrecho para Reconstrucción Nasal en Cancer de Piel. Cir. plást. iberolatinoam., 2016, 42(3):271-278.

7. Serracanta Domenech, J. et al. Reconstrucción Nasal Total: A Propósito un Caso. Cir. plást. iberolatinoam., 2007, 33(4):249255.

8. Menick, F.J. A 10 Year Experience in Nasal Reconstruction with Three Stage Forehead Flap. Plast. Reconstr. Surg. 2001, 109(6):1839-1855.

9. Burget, G.C. Discussion A 10 Year Experience in Nasal Reconstruction with Three Stage Forehead Flap. Plast. Reconstr. Surg. 2001, 109(6):1856-1861. 
